\title{
An Ethnographic Study of Near-Death Experience Impact and Aftereffects and Their Cultural Implications
}

\author{
L. Suzanne Gordon, Ph.D. \\ University of Maryland, College Park
}

\begin{abstract}
In this paper, I describe the research method and key near-death experience (NDE) aftereffects- and integration-related findings of my dissertation research study (Gordon, 2007), the first published near-death studies research project to use the ethnographic method. I compare my findings with those of a comparable sociological study (Sutherland, 1995), with emphasis on NDE aftereffects and integration issues related to what I identified as a previously unrecognized pattern of unmet, NDE-integration-related health-education and counseling needs. Finally, I explore the cultural implications of near-death and similarly transformative experiences and posit that actualizing the potential social-wellness value of these experiences to those who have had them and to their societies requires research and practice that adequately addresses experiencers' health-education and counseling needs.
\end{abstract}

KEY WORDS: near-death experiences, near-death experience aftereffects, near-death experience integration, health education, ethnography.

Speaking at the first annual conference of the American Center for the Integration of Spiritually Transformative Experiences (ACISTE) is a high point for me, as a researcher, a founding ACISTE board of direc-

L. Suzanne Gordon, Ph.D., is Lecturer in the Departments of Communication and American Studies at the University of Maryland and a founding member of the board of directors of the American Center for the Integration of Spiritually Transformative Experiences (ACISTE). This article is based on Dr. Gordon's presentation at the inaugural conference of ACISTE in October of 2012. The first phase of the study described in this article was supported by a grant from the International Association for NearDeath Studies and received an international graduate-student award for cross-cultural research in death and dying from the Association for Death Education and Counseling. Correspondence regarding this article should be sent to Dr. Gordon at the Department of Communication, 2130 Skinner Building, University of Maryland, College Park, MD 20742-7635; telephone: (301)405-8979, email: lsgordon@umd.edu. 
tors member, and a near-death experiencer. Helping to found ACISTE represents an important step in building on my research in ways that I hope will benefit not only the hundreds of near-death experiencers who contributed to the study I describe herein but also the millions more experiencers they represent in the United States alone. Because the research method I used is new to the field of near-death studies, I begin by introducing the research approach and study participants. Next, I compare the study's near-death-experience (NDE) integrationand aftereffects-related findings with those of Australian sociologist and near-death experiencer Cherie Sutherland's Ph.D. research study (1995). Finally, I discuss the cultural implications of these findings.

\section{About the Research Study}

As a contribution to the near-death studies literature, the study is significant in being the first project in the field to use the qualitative, phenomenological, participant-observation-field-work-based research method of ethnography. To make the study participants' lived experience, rather than the medical NDE model, the orienting, interpretative framework for the study, I used a life-history-narrative-based, or person-centered, ethnographic research approach (Caughey, 1995, 2006; Watson \& Watson-Franke, 1985). This practice situated the experiencers methodologically as the study's primary NDE experts. Consequently, my analysis and conclusions were based on more data and a wider range of data sets than are typical of previous studies. This result is in part because I spent a total of four years of full-time, face-to-face, intensive, ethnographic fieldwork with the 50 near-death experiencers whom I selected for participation from the hundreds of experiencers I met personally during the course of the study.

Previous near-death research studies more typically have used quantitative approaches shaped by the NDE research model for gathering and interpreting data for analysis. Such analyses may be based either on NDE accounts collected by previous researchers or on new accounts that often were obtained via mail or internet questionnaire responses from experiencers rather than by in-person interviews. This approach-even when experiencers have been interviewed in person, as is the case with the newer, prospective, more-rigorously-designed, in-hospital studies-generates NDE-related data heavily shaped by the medical NDE model (Kellehear, 1996) rather than by experiencers' own, self-generated experience accounts and interpretations.

This medical-model-based research approach has persisted in spite 
of the fact that—as this study was the first to reveal—the model does not meet the standards and criteria for health education and counseling research models, as is expected of models appropriate for use in the fields of hospice and death education and counseling (Corr, Nabe, \& Corr, pp. 20-25). As a result, rather than a body of healtheducation-and-counseling related knowledge about NDEs-that is, a body of near-death studies research literature focused on addressing the health needs as well as the potential social-wellness value of tens of millions of near-death experiencers-I found instead, in the study's literature review, a wide range of contested interests in NDEs and related phenomena among the field's contributors overall (Gordon, 2007, pp. 2-24). Further, I found a pattern of misinformation-and missing information - about NDEs in leading medical, health, and death-anddying education textbooks (Gordon, 2007, pp. 233-235) and, consequently, in the media and among the general public-experiencers included. It is not surprising, therefore, that this current study also revealed that experiencers' NDE-integration-related needs were often not recognized or disclosed as such, either immediately after the NDE or even decades later, whether in emergency rooms, therapists' and psychiatrists' offices, or hospice care.

In short, when I entered the field I found that the focus of research interest overall had been on explaining the meaning of NDEs (Kellehear, 1996)—from various, contesting, experts' perspectives-rather than on examining experiencers' own interpretations of the meaning of their experiences, or on addressing experiencers' health-, mentalhealth-, and counseling-related needs, or their collective potential social-wellness value. At the same time, it should be emphasized that, from the early days of the field, there have been some notable exceptions to this assessment, including investigator P. M. H. Atwater and researchers Raymond Moody and Kenneth Ring. In contrast, I was most interested in exploring the long-overlooked subject of how experiencers themselves, as the insider-experts on NDEs, would describe and interpret the meaning and significance of their NDEs and NDE aftereffects and in how they negotiated the lived, day-to-day process of NDE integration.

\section{Situating Myself}

A basic consideration of ethnographic research is how the researcher situates oneself with regard to the subject of the study. To situate myself in relation to my subject, my use of a near-death-experiencer- 
centered approach had roots in questions I had about the death of my mother just after she died in 1994, which I believed near-death studies literature would answer. This event was almost four decades since she had described, after having 'flat-lined' during emergency surgery following a miscarriage in 1956, a profound, after-death journey that, nearly two decades later, I would realize had been an NDE (Moody, 1975). As I began reviewing the research in the field, my interests were sharpened by two unexpected discoveries. First, I found very little focus on or systematic study of the impact and aftereffects of NDEs on experiencers' lives, let alone on their deaths, and on the lives of their significant others. In fact, more than a decade later, neardeath studies literature still has no answers for my original questions. Second, even more astonishing to me, I came across accounts of children's NDEs and, later, information about children's NDE aftereffects and their often arrested NDE-integration trajectories (Sutherland, 1995, pp. 230-235) that matched my own vivid, surgery-related experience, including its impact and aftereffects, that family and hospital records indicated had happened when I was 36 months old. Although the popularized medical NDE model-based literature misrepresented early data that indicated clearly that NDE elements such as a tunnel experience characterize only a portion of NDEs (Moody, 1975, p. 13; Ring, 1980), because I had concluded from that popularized literature that tunnels were essential elements of NDEs, and my experience did not include a tunnel, I had always been certain my experience could not have been an NDE.

My discovery during my research that I, myself, was a near-death experiencer undoubtedly complicated my role as researcher during fieldwork. In hindsight, I think my social role as a complete member of the near-death experienced culture I was studying during field work (Adler \& Adler, 1987, pp. 67-84) — especially my NDE-newcomer befuddlement and thus my personal interest in what the study participants could tell me about NDEs and NDE aftereffects-also helped me during research. It helped me to gain the trust of the study participants and, thereby, to learn first-hand about the kinds of NDEintegration challenges experiencers encounter.

These circumstances also informed my reformist qualitativeresearch ethics and approach (Denzin \& Lincoln, 2006), which made me accountable to near-death experiencers as the primary beneficiaries of my work: The study was meant to be for the experiencers whose lives and experiences I was studying, not simply about them. Within an interdisciplinary, American Studies scholarly context, my 
interpretive tools included social-construction and identity-alteration theories (Berger, 1963, pp. 54-65; Berger \& Luckmann, 1966). More relevant to this discussion, thanks to mentoring by Daniel Leviton, death-education pioneer and founding president of the Association for Death Education and Counseling, the study's analysis was critically informed by situating experiencers and their NDEs within the context of human health and wellness studies, particularly death education and counseling studies (e.g., Corr et al., 1997, DeSpelder \& Strickland, 1999; De Vries, 1999; Leviton, 1991; Moller, 1996).

\section{Research Participants, Approach, and Selection}

During fieldwork, I relied primarily on my own social networks to locate near-death-experienced research participants. This approach is the traditional one that ethnographers use to locate and engage potential fieldwork informants, whether within their own or in other cultures. Because of concerns about the impact of near-death-studies acculturation on experiencers' NDE accounts, I met and engaged 29 of the 50 near-death experiencers in the study-and easily could have engaged all 50-simply by communicating my research interest to my social networks and contacts, outside of the context of near-death studies environments or organizations. For instance, I met one of the primary informants in line at a local health food store. For purposes of contrast, I engaged the other 21 through contact with the International Association for Near-Death Studies (IANDS), whose membership has always been open to and composed of near-death experiencers as well as near-death studies researchers.

The approach I found most useful in describing the study to contacts and potential research participants was to explain that I was "interested in learning about the lives of people who have had experiences like near-death experiences." I used this phrasing because I had been surprised to discover, from my initial contact with my first study participant, that experiencers were not necessarily certain, or even aware, of their experiences as near-death experiences, nor knowledgeable about near-death studies. This phrasing allowed me to engage informants whether or not they previously had identified their experiences as NDEs. This practice was not typical of most previous retrospective research studies, in which research have typically drawn subjects from self-identified near-death experiencers willing to serve in near-death studies research projects. I suspect this factor may make them unrepresentative of the general population of NDE survi- 
vors, based on this study and on what I know about the demographics of near-death-studies-acculturated near-death experiencers. For example, experiencers who have connected with IANDS would seem to comprise only a small fraction of the general population of experiencers. This point may be important, if my observations are correct, because IANDS-affiliated experiencers do not seem to be demographically representative of the general population in other respects, for instance, with regard to ethnic diversity. My approach made perhaps 29 of the 50 study participants atypical of previous near-death studies subject groups, but only in their having little or no knowledge of or interest in near-death studies. Otherwise, the criteria for selecting the 50 experiencers for the study made these 50 individuals comparable to previous research groups of near-death experiencers of the same size, including those in Sutherland's study (1995).

Of the 50 study participants, 15 were males and 35 females, with an age range of 20 to 70 years. Eight of the 50-including 2 of the 10 experiencers in the core-participants group-were of African-American descent. One was of Native North American descent, 1 was of Iranian descent, and the other 40 , including 8 of the 10 core participants, were of Eastern or Western European descent. Of four non-U.S. citizens in the background groups, two were Australian and two were Canadian.

Tables 1 and 2 provide some basic information for comparing the NDE-related data I used to select the study's 50 near-deathexperiencer-participants and to assign their membership in the core group $(n=10)$ or the background group $(n=40)$ and, within the latter group, either IANDS $(n=20)$ or non-IANDS $(n=20)$ background subgroup. All participants completed the Near-Death Experience Scale (NDE Scale; Greyson, 1983). The NDE Scale is the most widely used instrument to assess the presence and depth-number and intensity of elements - of an NDE. The Scale has established validity and reliability and has four components:

a cognitive component, including time distortion, thought acceleration, life review, and sudden understanding; an affective component, comprising feelings of peace, joy, and cosmic unity and an experience of a brilliant light; a paranormal component, including enhanced vision or hearing, apparent extrasensory perception, precognitive vision, and an out-of-body experience; and a transcendental component, comprising encounters with an apparently unearthly realm, a mystical being, and visible spirits and a barrier or point of no return that, had the subjects crossed it, would have precluded their return to life. (Greyson, 1985, pp. 967-968) 
Table 1 Core Participants' NDE-Related Information

\begin{tabular}{lllllll} 
& \multicolumn{2}{c}{ NDE Scale } & & & \multicolumn{2}{c}{ NDE } \\
\cline { 2 - 3 } \cline { 6 - 6 } $\begin{array}{l}\text { Participant } \\
\text { Pseudonym }\end{array}$ & \multicolumn{1}{c}{$\begin{array}{c}\text { Highest } \\
\text { Component }\end{array}$} & $\begin{array}{c}\text { Total } \\
\text { Score }\end{array}$ & $\begin{array}{c}\text { Birth } \\
\text { Year }\end{array}$ & $\begin{array}{c}\text { Age in } \\
\text { years }\end{array}$ & \multicolumn{1}{c}{ Circumstance } \\
\hline Neil & Cognitive & 29 & 1949 & 27 & Shooting \\
Alicia & Cognitive & 26 & 1950 & 35 & Car accident \\
Gwen & Cognitive & 23 & 1946 & 38 & Train wreck \\
Nagual & Cognitive & 23 & 1946 & 54 & Acute seizures, \\
Jenny & Transcendental & 18 & 1943 & 49 & Pancreatitis and diabetic \\
& & & & & complications, in I.C.U. \\
Kelsey & Affective & 17 & 1937 & 41 & Fall down elevator shaft \\
Chris & Transcendental & 17 & 1950 & 34 & Thrown from horse \\
John Paul & Cognitive & 17 & 1985 & 16 & Pneumonia \\
Eric & Affective & 17 & 1943 & 20 s & Drowning \\
Camille & Affective & 12 & 1960 & 16 & Hemorrhage \\
\hline
\end{tabular}

Table 2 Summary of NDE-Related Selection Criteria Data for Background Groups

Non-IANDS- IANDS-

Affiliated Group Affiliated Group

\section{Total number}

Criteria not met:

None (all criteria met)

Age: Younger than 16 years at NDE

\section{Total reporting $N D E s$}

No life-threatening event

NDE Scale score below 7 and no lifethreatening event

Total reporting other, 'near-death-like experiences'
10

5

3

20

20

\begin{tabular}{cc} 
& \\
15 & 14 \\
2 & 4 \\
3 & 2 \\
& \\
\hline 5 & 6
\end{tabular}


For possible assignment to the core group, a participant had to have been at least age 16 years at the time of surviving an actual life-threatening episode accompanied by a subjective experience that scored at least 7 on the NDE Scale (Greyson, 1983). All core group members met all criteria, whereas only about half of experiencers in either background subgroup met all criteria. From among all approximately 30 participants who met all criteria, I assigned 10 to the core group because I considered them representative of the entire 50 -person study group both demographically and in terms of the circumstances and the range of NDEs they reported and for their geographic accessibility and availability to participate in the requisite extensive interview sessions and other ongoing fieldwork interactions over periods that ranged from three months in one case to more than a decade in six cases.

The core group was comprised of 9 of the 29 near-death experiencers I engaged on my own and-simply for contrast- 1 of the 21 experiencers I met through IANDS. For the study, I drew most extensively on the core group members' complete, self-generated, life-history narratives for quotes and analysis. From these accounts, I selected seven for inclusion in full in the original study write-up, to illustrate the range of experiences I encountered during the four years of fieldwork.

I assigned participants to background group membership to represent, collectively, the range of NDE and NDE-like accounts I heard from experiencers at IANDS gatherings and found in the near-death studies literature. Non-IANDS background subgroup members were 20 of 29 participants I had met on my own, and IANDS background subgroup members were 20 of an unknown total I had met through IANDS; several among this latter group had played key leadership roles in IANDS.

The question I asked, to elicit participants' self-generated life stories was: "If you were going to write or tell someone your life story, how would you organize it into chapters?" (Caughey, 2006, pp. 28-29). In addition to copious field notes on observations, interactions, and discussions with all 50 near-death experiencers in the study and many other potential participants, the multiple, audio-recorded interviews with the 10 core informants collectively generated 1,167 single-spaced, typed pages of transcribed life history narrative material for analysis. The findings and comparisons described below are drawn mainly from this material and supplemented, where noted, with information on the individuals in the background groups. 


\section{Overview of Key Study Findings and Comparisons}

As mentioned, to contextualize the study's findings of the NDE aftereffects of the study participants, they were compared in the dissertation with those of the 50 experiencers who participated in Sutherland's sociological study (Sutherland, 1995). Her study tested and supported a substantial body of previous evidence-mirrored by this study's findings (Gordon, 2007, pp. 179-229) —which pointed to a striking pattern of commonly reported, although still under-researched, NDE aftereffects and NDE integration difficulties. Her study also offered a model for describing different $N D E$ integration trajectory types (Sutherland, 1995, pp. 205-237), based on her study of the process by which, when integration is not "blocked" or "arrested" (Sutherland, p. 219) by various life circumstances, experiencers come to align their lives with what they learned during their NDEs:

There comes a time when the experiencers recognize (often in retrospect) that their NDE is now a part of their lives, congruent not only with their attitudes but also their actions. . . . [It] can be facilitated or hindered by a wide variety of social interactions. . . . [Integration is] an ongoing dynamic process constantly being negotiated and managed by the individual within a particular social, intellectual, historical context ... [and it] can encompass many transitions along its path. (Sutherland, 1995, p. 206)

Although not discussed here, her findings related to NDE integration trajectory patterns were also supported by this study's findings (Gordon, 2007, pp. 215-229).

This section focuses on this study's findings of the most commonly reported types of NDE aftereffects and integration-related difficulties, organized into seven categories based on Sutherland's categories (1995, pp. 71-191).

Following each aftereffects- or integration-issues category heading below, the set(s) of numbers in parentheses refer, respectively, to the number of this study's 10-person core-informants group affected and, where known, to the number of subjects affected in the backgroundinformants groups. For each of these categories, except where indicated, at least $75 \%$ of Sutherland's subjects were affected. Note that, because this study's data were derived from participants' selfgenerated life history narratives, which did not necessarily include the same kinds of information, comparison data for participants in the background groups are not known for all categories. Likewise, Sutherland's sociological grounded-theory-based interview protocol allowed 
experiencers' responses to affect her decision to ask, or not ask, particular sets of follow-up questions (or to add new ones in some cases), so, within the discussion of each category below, the total number of her subjects' responses used for comparison may vary.

\section{NDE Impact: Identity-Reality Context Shift (8/10, 46/50)}

The NDE has been validated in medical-scientific terms as an "anomalous," but "profound, psychological event" (Greyson, 2004, pp. 604605). Whereas diverse, indigenous knowledge systems and world wisdom traditions attribute profound social, or real-world, meaning, as well as personal significance, to such sacred death-and-rebirth experiences, the material worldview of contemporary Western culture does not. For the majority of experiencers in this study, scores of their comments indicated that the impact and aftereffects of NDEs are seen to follow naturally from, and provide more evidence of, experiencers' first-hand experience of the reality of the newly recognized dimensions of the sacred world revealed on the other side of physical death-not necessarily 'otherworldly' dimensions, but previously unknown oneswhich prevailing Western culture denies are real. Sutherland (1995) identified this profound reality/identity context shift or "ontological shift" (p. 193) as the defining feature marking activation of the NDE integration process.

One of this study's background participants, cultural anthropologist Patrick Gallagher, in an early journal article now considered a classic in near-death studies literature, described the context-shift that marks the activation of NDE integration in ethnographic terms as the culture shock that he experienced at the onset of his NDE and the counter shock he experienced afterward (Gallagher, 1982). Many comments by study participants supported Gallagher's understanding of the phenomenological reality and subsequent real-world impact of an NDE. From an ethnographic perspective, NDE integration may be seen as a process strongly influenced or even determined by how experiencers' previously held belief-and-value systems and those of the newly discovered 'reality-context' intersect, and are negotiated, within experiencers' individual social worlds. Eight of the core-participant accounts and $92 \%$ of all accounts included reference to a context shift: a global shift in awareness of one's identity and reality. 


\section{NDE Disclosure and Disclosure-Context Difficulties $(10 / 10,50 / 50)$}

A theme in Sutherland's (1995) findings related to difficulties in discussing NDEs involving the ineffability and the unknown nature of the experiences as well as the reactions of others. This is a critical problem because, as she found, disclosure is necessary to the integration process-so much so that it typically requires some restructuring of the experiencer's social disclosure-context. For instance, three people in her study who received initial negative responses did not relate their experiences to anyone else for years. Thirteen others did not discuss their experiences with anyone for years and then remained very selective. Three had never talked to anyone before Sutherland (1995, pp. 71-77); this was the case with at least two of this study's core-group participants.

Like Sutherland's (1995) subjects, 100\% of this study's participants described NDE disclosure-related problems that collectively demonstrated what I identified as a previously-unrecognized, pervasive pattern of NDE-integration related, unmet health-education-andcounseling needs. These included significant difficulties and problems related to NDE identification or interpretation; NDE disclosure; and the lack of appropriate, accessible, education and counseling resources and support for the NDE integration process overall (Gordon, 2007, pp. 190-240). Most basically, the need is for near-death experiencers and their professional medical and mental health care providers to have an NDE model and research agenda that meet health- and death-education standards. Experiencers and their healthcare providers also need appropriate and accessible knowledge and expertise so they are able to identify NDEs as NDEs and recognize NDE aftereffects as NDE aftereffects. Meanwhile, whether in health-care, counseling, family, or other contexts, both core and background study participants characterized the majority of others' reactions to their NDE disclosure statements negatively: as dismissive, uninformed, or otherwise problematic. The problems study participants described can be grouped into the following five categories of NDE-disclosure-related difficulties:

- Inappropriate NDE-disclosure reactions from family and medical professionals. Five of the core participants described negative NDE-disclosure responses from family members, and three described dismissive or uninformed reactions from medical professionals. 
- NDE disclosure problems in mental health and counseling contexts. From the mid-1980s to 2005, five of the core participants sought psychiatric, family therapy, or other counseling help to deal with characteristic NDE aftereffects- or other integration-related issues. These issues were neither identified as such by any of them nor addressed as such by any of their mental health professionals. These included immediate NDE impact issues, such as euphoria in one case that led to an erroneous diagnosis of bipolar disorder; relationship problems; and depression.

- NDE recognition and NDE model problems. At the time of their experiences,-eight of the core-group experiencers were unaware that their experiences were NDEs. At the start of the study, only of these were absolutely certain of their experiences as NDEs, and an additional three had some understanding of their experiences as NDE-like. At the conclusion of the study, despite unequivocal scores on the NDE Scale, two of these eight core experiencers still remained doubtful that their experiences actually had been NDEs because elements of their NDEs did not match the NDE model as they understood it.

- Resistance to NDE label and use of alternate labels. Nine of the core informants' accounts illustrated a preference for some label other than near-death experience or NDE for their experience. Even among core informants who had identified their experiences as NDEs, only one (Jenny, the only IANDS-affiliated core informant), used that label as her preferred one. Five of the other nine core participants avoided using the label at all, and the remaining four used it in ways that problematized the NDE label. For example, Kelsey preferred the term "spiritual revelation," and Chris at first wondered if she had suffered "brain damage," or was "crazy." Gwen invariably referred to either "the train wreck" or "when I died in the train wreck" to label her experience; or else, in comparison with what she termed our "world of shadows," she referred to her experience in the "world of light." In fact, in 1995, when I first used the term NDE to refer to her experience, her response was, "What's an NDE?" Neil rejected the NDE label because he had heard himself pronounced dead; therefore, he considered his NDE to have been an after-death experience, and his preferred label for it was "shamanic death and rebirth."

- Near-death studies and IANDS as problematic NDE integration or disclosure contexts or resources. The accounts of $98 \%$ of the study's total participants, including nine of the core informants, indicated either that they were unaware of IANDS and the field of near-death studies or that they found these resources problematic. The kinds of NDE-disclosure issues described above may help explain why so many of the study participants, including 20 of the 21 IANDSaffiliated informants, seem to have found the clinical NDE model, neardeath studies literature, and IANDS problematic resources for NDE 
integration - when they were aware of these resources at all. For nine core participants and all 20 in the non-IANDS-affiliated background group - that is, for all 29 of the non-IANDS-affiliated participantsthere was no indication that either near-death studies experts or IANDS played any role as NDE disclosure-contexts or integration resources. Further, many participants in the core and both background groups made comments that were explicitly critical of medical-scientific neardeath studies research and expertise. Within IANDS, experiencers' initiatives have led to experiencers-only sessions and meeting spaces at IANDS conferences,-as well as to separate retreats for experiencersonly (Stout, Jacquin, \& Atwater, 2006, pp. 49-62) - a development that, paradoxically, illustrates IANDS' responsiveness to NDErs' needs.

\section{Changes in Fears of and Attitudes about Death $(10 / 10,50 / 50)$}

The percentage of those in Sutherland's study (1995) who reported having no fear of death changed from $19 \%$ pre-NDE to $98 \%$ post-NDE (p. 81). Regardless of previous beliefs, $100 \%$ of this study's participants expressed new or increased awareness of the self's survival of physical death and loss of fear of death. Twenty of Sutherland's 50 subjects reported an increased interest or involvement in work with the dying (pp. 91-93) as did two of this study's core participants. Also, $14 \%$ of this study's background participants had become hospice volunteers prior to having identified their experiences as NDEs.

\section{Changes in Religious Beliefs, Values, Interests, Sense of Spirituality, Life-purpose (9/10)}

The majority of Sutherland's (1995) and this study's participants indicated that after their NDEs they became more spiritually focused and less concerned with religious doctrine. The percentage of her subjects who indicated no religious affiliation jumped from $46 \%$ pre-NDE to 86\% post-NDE (Sutherland, 1995, pp. 94-97). With the exception of the one agnostic among this study's core-participants, the other nine also described changes in interests, beliefs, and values related to a heightened sense of spirituality. That is, they described spirituality in other than narrow, religious-doctrine-based terms-as growth in the ability to love and serve others, for instance, or as being central to the meaning of life and to the purpose or mission of their own lives. Of the five who kept or initiated a religious affiliation, all described a heightened interest in learning about various spiritual, religious, and 
other wisdom traditions, and a greater understanding that all paths to spiritual growth have value, that no one path is the best or only one. "Overall," as Sutherland (1995) observed, "there is a feeling [of] . . an ongoing direct contact with God or a Higher Power that requires no mediation" (p. 110).

\section{Psychic/Paranormal Experiences, Abilities (8/10)}

Increased incidence of a wide range of psychic or paranormal experiences and abilities, from out-of-body experiences to the ability to heal others, were found in Sutherland's study (1995), with more than 85\% reporting incidence or increased incidence of post-NDE experiences of such phenomena as telepathy, precognition, and intuition (pp. 112135). Similarly, eight of this study's core-group participants reported such phenomena either beginning or significantly intensifying after their experiences. All 10 core informants described such phenomena as part of their NDEs, and three of them reported observations during their NDEs that were later confirmed by others. Eight of 10 core participants expressed precognitive awareness or intuitive knowledge of future events or shifts of global significance. Collectively, as a result of these types of experiences, they described the need for profound changes in the understanding of existence and the self and, as a result, in cultural beliefs, values, and priorities.

\section{Life Direction/Priority Shifts and Difficulties $(\mathbf{1 0 / 1 0}, \mathbf{5 0 / 5 0 )}$}

All $100 \%$ of study participants reported shifts in their sense of life direction and priorities as well as difficulties associated with these shifts. At least $60 \%$ percent of the respondents in both Sutherland's (1995, pp. 136-149) and this study reported each of the following: increased sense of self-worth, increased sense of spiritual mission or life purpose, increased desire to help others and decreased self-interest, increased sense of compassion and empathy, increased ability to express love, increased insight into others' problems and social or planetary problems, and decreased concern for others' opinions of oneself (Gordon, 2007, pp. 208-212). In both studies, life direction and priority shifts were connected to a range of difficulties in post-NDE adjustment. Such NDE integration difficulties have also been found in P. M. H. Atwater's investigations (e.g., Atwater, 1994, pp. 127-235). Sutherland (1995) found, for instance, that 23 of 34 subjects reported 
post-NDE strains in relationships with a spouse or partner (p. 151). Similarly, six of this study's core participants divorced or ended primary-partner relationships after their NDEs for reasons they identified as arising from or related to their NDEs.

\section{Deepened Awareness of, Shifted Attitudes toward Social Issues (9/10)}

In Sutherland's study (1995), 35 of 40 people who responded to questions in this topic area reported increased interest in social issues (Sutherland, 1995, p. 182). Similarly, the comments of nine of this study's core participants included astute, deeply-felt criticisms of Western culture's materialist worldview, with its attendant successdriven values and norms. For instance, one of them (Neil) articulately described the social construction of the "inadequate self," along with its economic implications, and how his NDE had helped him to see through it.

\section{Discussion: Limitations and Cultural Implications}

Two limitations of this study are, first, its relatively small size, and, second, that it is a retrospective study and can be criticized on the same grounds that have been used to criticize other such studies in the field. To address these limitations, the ethnographic method could be used in future, prospective studies involving larger groups of near-death experiencers. Also, because ethnographic researchers rely on their own social networks to find and engage fieldwork informants, the use of the ethnographic method by multiple researchers would mitigate the potential for unrecognized bias to influence ethnographic researchers' selection of study participants.

With these limitations in mind, the findings from this study indicate that the NDE is very often a life-changing experience. NDEs may seem otherworldly in relation to the consensual, material reality of contemporary society. But unlike vivid dreams or hallucinations, NDEs and their aftereffects are so real for experiencers that, like the cultural immersion of ethnographic field work in distant and culturally alien societies, NDEs often cause the same sort of culture shock and counter shock that anthropologist and NDE survivor Patrick Gallagher described (Gallagher, 1982).

Moreover, as can be illustrated by historical and anthropological accounts from diverse cultures, transformational experiences such 
as NDEs, and the lives of those who have them, have had profound and widespread social and historical significance (Caughey, 1984, pp. 237-240). Before recent advances in trauma-related and other medical technology, living for very long after having an NDE is thought to have been so much less common than it is now that in Western societies they would have been referred to as deathbed visions (Zaleski, 1987). But even when it may have been much less common to recover and live on for decades after an NDE, such profoundly-life altering, otherworldly journeys typically have marked the lives of individuals of diverse cultures who have initiated all of the world's major religions and wisdom traditions as well as other widespread social revitalization movements (Wallace, 1956). A 1992 Gallup poll found that 13 million adults in the United States, or 5\% of the population, had experienced NDEs; it has recently been estimated that, every day, approximately 774 more adults in the U.S. survive an NDE (Long, 2013).

Considering that individuals who have had NDEs and similarly lifetransforming experiences have had such profound impact throughout history and across the world's diverse cultures, what might be the potential social-wellness value of an estimated American population of 13 million experiencers? Particularly given the ways that near-death experiencers' accounts 'speak back' to both medical science and religious fundamentalisms, what kind of cultural influence might they, collectively, exert? From the cultural perspective, this issue would seem to be a significant, because these individuals can be seen to comprise a substantial, if still all-but-invisible minority population whose members share a remarkably atypical and potentially highly socialwellness-enhancing set of cultural values (Leviton, personal communication, April 10, 2009).

Although diverse disciplines-including medicine, psychiatry, and psychology-are represented in near-death studies, there has been little focus overall on addressing the health education, mental health, and counseling needs of experiencers. In fact, before initiation of a recent ACISTE survey study, there had never been a formal study designed to assess what experiencers' needs are. The study described in this article addressed the health education and counseling needs of a population of millions, and perhaps tens of millions, of near-death experiencers. In approaching it, I recognized the potential socialwellness value of near-death experiencers' collective visibility as agents, among many others, of a sacred, multidimensional worldview, one that is linked not only to the worldviews of diverse indigenous 
knowledge systems and non-Western wisdom traditions but also to the multidimensional worldview of quantum physics.

One example of the importance of the collective cultural visibility (or invisibility) of near-death experiencers in the contemporary Western world is that, to quote Sutherland (1995), "the implications of having a [socially visible] population of people with no fear of death are significant in a society such as our own, fraught as it is with death denial" (p. 242). When people lose the fear of death it becomes possible, she argued, "to engage in meaningful relationships with the dying, to abandon immortality projects ... [and] vehicles such as money [and] fame. . . as ultimately illusory" (p. 242). Most important, she wrote, "such crucial changes in attitude provide a fundamental challenge to the widely accepted norms of Western society" (p. 242):

When it is realized that millions of people worldwide have had this experience ... it can be seen that the potential force for positive social change is enormous. These people know that death is not the end.... Almost all of them are changed by the experience, and through their interactions with others these changes move beyond the personal, beyond the lives of individual experiencers into the social realm, presaging a profound transformation of great benefit to society as a whole. (Sutherland, 1995, p. 242)

However, as psychologist Kenneth Ring (1998) has observed:

Most NDErs have a difficult time coming to terms with their experience, and the process of its integration into their lives. This may take a long time, and, certainly, in some cases, it does not occur at all. Longstanding relationships may be strained to and beyond the breaking point, marriages collapse, misunderstandings are common, and periods of painful introspection and even depression are not rare. The NDE, as we have seen, tends to turn a person's life topsy-turvy, and the radical reorientation and personal courage to live out the truth of one's NDE may be very taxing indeed, both to the NDEr and his or her family and friends. . . . Most people, when they hear . . . or read of NDEs, feel a certain amount of envy, wishing that they too could have the experience (without, to be sure, having to go to the trouble of nearly dying for the privilege). But if they could . . . they would soon realize that the NDE is often a mixed blessing and may continue to extract a high cost in suffering from the individual's life. (pp. 32-33, 35)

Given the size of the near-death-experienced population, it matters, therefore, whether and how effectively research, education, and counseling resources meet the NDE-related health-education and counsel- 
ing needs of experiencers, their health-care and counseling providers, and their loved ones. It matters whether or not a global community of tens of millions of near-death experiencers has the support needed to allow experiencers to become visible to themselves and, collectively, visible to each other and to society-at-large.

\section{References}

Adler, P. A., \& Adler, P. (1987). Membership roles in field research: Qualitative research methods series 6 . Newbury Park, CA: Sage.

Atwater, P. M. H. (1994). Beyond the light: The mysteries and revelations of neardeath experiences. New York, NY: Avon.

Berger, P. (1963). Invitation to sociology. New York, NY: Anchor.

Berger, P., \& Luckmann, T. (1966). The social construction of reality: A treatise in the sociology of knowledge. New York, NY: Doubleday.

Caughey, J. (1984). Imaginary social worlds: A cultural approach. Lincoln, NE: University of Nebraska.

Caughey, J. (1995). Individual identity and multiple cultures: Teaching difference through life history. Paper presented at the Annual Meeting of the American Studies Association. Pittsburgh, PA.

Caughey, J. (2006). Negotiating cultures and identities: Life history issues, methods, and readings. Lincoln, NE: University of Nebraska.

Corr, C. A., Nabe, C. M., \& Corr, D. M. (1997). Death and dying, life and living (6th ed.). Belmont, CA: Wadsworth.

Denzin, N., \& Lincoln, Y. S. (2005). The Sage handbook of qualitative research (3rd ed.). London, England: Sage.

DeSpelder, L.A., \& Strickland, A. S. (1999). The last dance: Encountering death. Mountain View, CA: Mayfield.

De Vries, B. (1999). End of life issues: Interdisciplinary and multidimensional perspectives. New York, NY: Springer.

Gallagher, P. (1982). Over easy: A cultural anthropologist's near-death experience. Anabiosis, 2(2), 140-149.

Gordon, L. S. (2007). Field notes from the light: An ethnographic study of the meaning and significance of near-death experiences (Unpublished doctoral dissertation). University of Maryland, College Park, MD.

Greyson, B. (1983). The near-death experience scale: Construction, reliability, and validity. Journal of Nervous and Mental Disease, 171(6), 369-375.

Greyson, B. (1985). A typology of near-death experiences. American Journal of Psychiatry, 142(8), 967-969.

Greyson, B. (1990). Near-death encounters with and without near-death experiences: Comparative NDE Scale profiles. Journal of Near-Death Studies, 8(3), 151-161.

Greyson, B. (2004). Near-death experiences. In The concise Corsini encyclopedia of psychology and behavioral science (3rd ed.; pp. 604-605). Hoboken, NJ: John Wiley \& Sons.

Kellehear, A. (1996). Experiences near death: Beyond medicine and religion. New York, NY: Oxford University Press. 
Leviton, D. (Ed.). (1991). Horrendous death and health: Toward action. New York, NY: Hemisphere.

Long, J. (2013). How many NDEs occur in the United States every day? Retrieved from http://www.nderf.org/NDERF/Research/number_nde_usa.htm.

Moller, D. W. (1996). Confronting death: Values, institutions, and human mortality. New York, NY: Oxford University Press.

Moody, R. A. (1975). Life after life. Covington, GA: Mockingbird Books.

Ring, K., \& Valarino, E. E. (1998). Lessons from the light: What we can learn from near-death experiences. Covington, MA: Perseus Books.

Stout, Y. M., Jacquin, L. A., \& Atwater, P. M. H. (2006). Six major challenges faced by near-death experiencers. Journal of Near-Death Studies, 25(1). 49-62.

Sutherland, C. (1995). Reborn in the light: Life after near-death experiences. New York, NY: Bantam.

Wallace, A. F. C. (1956). Revitalization movements. American Anthropologist, 58(2), 406-409.

Watson, L., \& Watson-Franke, M. (1985). Interpreting life histories: An anthropological inquiry. New Brunswick, CT: Rutgers University Press.

Zaleski, C. (1987). Otherworldly journeys. New York, NY: Oxford University Press. 